\title{
A search for $s$-process elements in extremely metal-poor halo planetary nebulae
}

\author{
Masaaki Otsuka ${ }^{1}$, Akito Tajitsu $^{2}$, Hideyuki Izumiura $^{3}$, \\ and Siek Hyung ${ }^{4}$ \\ ${ }^{1}$ Space Telescope Science Institute, \\ 3700 San Martin Dr., Baltimore, MD 21218, U.S.A. \\ email:otsuka@stsci.edu \\ ${ }^{2}$ Subaru Telescope, NAOJ, \\ 650 North A'ohoku Place, Hilo, Hawaii 96720, U.S.A. \\ ${ }^{3}$ Okayama Astrophysical Observatory (OAO), NAOJ, \\ Kamogata, Okayama 719-0232, Japan \\ ${ }^{4}$ School of Science Education (Astronomy), Chungbuk National University, \\ 12 Gaeshin-dong Heungduk-gu, CheongJu, Chungbuk 361-763, Korea
}

\begin{abstract}
We have performed deep high-dispersion spectroscopy to examine enhancement of $s$-process elements in the exremely metal-poor $([\mathrm{Ar} / \mathrm{H}] \sim-2.1)$ halo planetary nebulae $\mathrm{H} 4-1$ and BoBn1 using the 8.2-m Subaru telescope/High-Dispersion Spectrograph (HDS). We have detected several emission lines of s-process elements in $\mathrm{H} 4-1$ and $\mathrm{BoBn} 1$, and we have found that the enhancement of heavy $s$-process elements in these objects is comparable with that in $s$ process enhanced CEMP stars with $[\mathrm{Fe} / \mathrm{H}]>-2.5$. The C- and N-rich abundances of $\mathrm{H} 4-1$ and BoBn1 might be explained by binary evolution models. We have detected 5 fluorine lines in BoBn1. The re-estimated $\mathrm{F}$ abundance using these lines is $[\mathrm{F} / \mathrm{H}]=+1.4 \pm 0.1$.
\end{abstract}

Keywords. (ISM:) planetary nebulae: individual (H4-1, BoBn1), stars: evolution

\section{Introduction}

Currently, over 1,000 objects are regarded as planetary nebulae (PNe) in our Galaxy, while 14 of them have been identified as halo members from their location and kinematics. Of them, 5 objects are known as $\mathrm{C}$ - and N-rich $([\mathrm{C}, \mathrm{N} / \mathrm{O}]>0)$ halo $\mathrm{PNe}$ with $[\mathrm{Ar} / \mathrm{H}]<-1.7$. K648 (in M15), H4-1, and BoBn1 are C- and N-rich halo PNe with $[\mathrm{Ar} / \mathrm{H}] \sim-2.1$. The progenitors of halo PNe are thought to be $\sim 0.8 M_{\odot}$ stars. These C- and N-rich halo PNe, however, show signatures that they have evolved from massive progenitors.

For example, these halo PNe can become N-rich, but not C-rich, if they have evolved from single $\sim 0.8 M_{\odot}$ stars. To become C-rich PNe, the third dredge-up must take place in the late AGB phase. However, the third dredge-up takes place in stars with initial masses $>1.2-1.5 M_{\odot}$. Also, current stellar evolutionary models predict that the postAGB evolution of a star with an initial mass $\sim 0.8 M_{\odot}$ proceeds too slowly for a visible PN to be formed. Unless these issues are resolved, we will not be able to take full advantage of halo PNe as a proof of low-mass star evolution and the Galactic chemical evolution.

How these progenitor stars became $\mathrm{C}$ and N-rich halo PNe? To answer this key question, we have been observing these halo PNe using the 8.2-m Subaru telescope/HighDispersion Spectrograph (HDS) and collecting archival data and carefully analyzing spectra of these objects. Our recent research reveals that $[\mathrm{C} / \mathrm{Fe}]$ and $[\mathrm{N} / \mathrm{Fe}]$ abundances of K648, H4-1, and BoBn1 are compatible with those of carbon-enhanced metal poor (CEMP) stars (Otsuka et al. 2008a). Interestingly, BoBn1 is the most fluorine rich among 

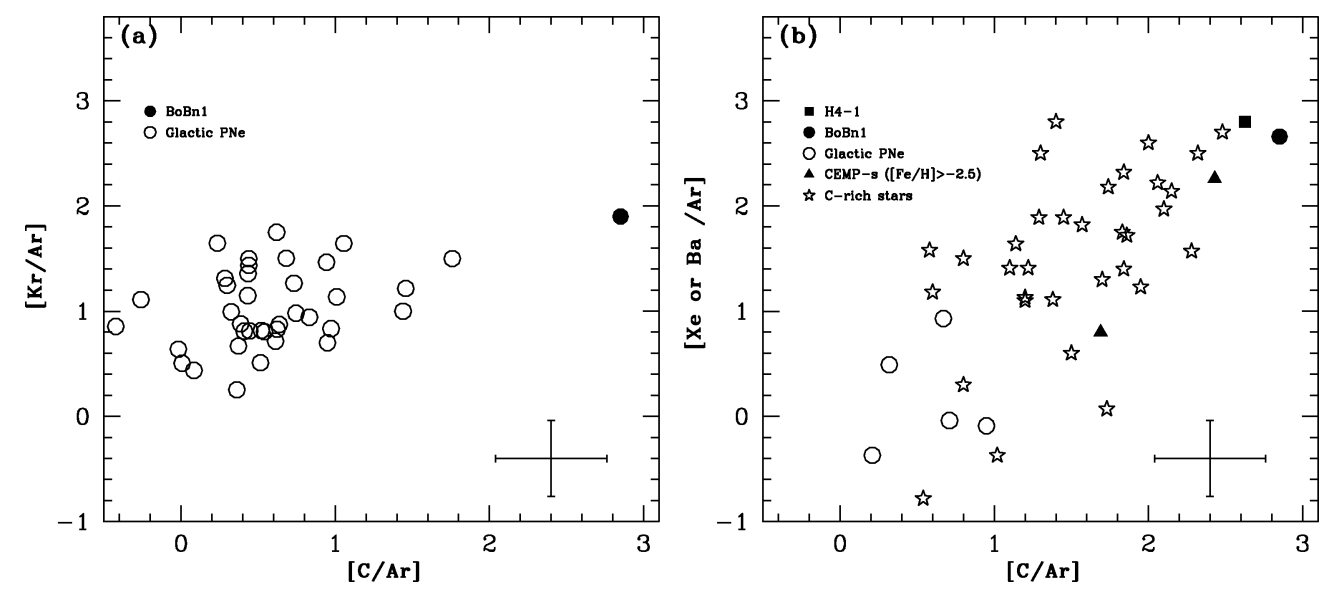

Figure 1. (left panel) [Kr/Ar]-[C/Ar] diagram. Galactic PNe: the data of 37 objects taken from Sterling \& Dinerstein (2008). (right panel) [Xe or Ba/Ar]-[C/Ar] diagram. Galactic PNe: taken from Sharpee et al. (2007). CEMP-s and C-rich AGB: taken from the SAGA data base (Suda et al. 2008).

F-detected PNe, and its F-abundance is comparable with the $s$-process enhanced CEMP star (CEMP-s) HE1305+0132 (Otsuka et al. 2008b). These C-, N-rich halo PNe might share their origins with CEMP-s stars. If these halo PNe had evolved from binary stars such as CEMP-s stars, $s$-process element might be enhanced as well as C and N. To examine enhancement of $s$-process elements and verify our hypothesis, we have performed deep HDS spectroscopy for H4-1 and BoBn1. In this paper, we present our recent results of chemical abundance analysis of these objects.

\section{Observations \& results}

We have secured the spatially $(<0$.' 6 seeing) and spectrally $(R>33,000)$ highestresolution spectra with high $\mathrm{S} / \mathrm{N}$ ( $>40$ at the nebular continuum), and detected $>300$ emission lines for each object. We solved level populations for a multi-level (2-30 levels) atomic model using our codes and estimated $>14$ elemental abundances. The remarkable findings are as follows: (1) In H4-1 and BoBn1, several emission lines of $s$-process elements such as krypton ( $\mathrm{Kr}, Z=36$ ), rubidium (Rb, $Z=37$ ), xenon (Xe, $Z=54$ ), and barium (Ba, $Z=56$ ) are detected. The detections of these elements are for the first time. (2) H4-1 and BoBn1 are $s$-process element rich PNe (Fig. 1). The [Kr, Xe, Ba/Ar]-[C/Ar] diagrams indicate that $\mathrm{C}$-rich objects are also $s$-process element rich. This is consistent with nucleosynthesis theory for low-intermediate mass stars. The enhancement of heavy $s$-process elements in H4-1 and BoBn1 is comparable with that in CEMP- $s$ stars. The C- and N-rich abundances of these PNe might be explained by binary evolution models. (3) In BoBn1, 5 fluorine lines are detected. The re-estimated F abundance using these lines is $[\mathrm{F} / \mathrm{H}]=+1.4 \pm 0.1$.

\section{References}

Otsuka, M., Izumiura, H., Tajitsu, A., \& Hyung, S. 2008a, Origin of Matter and Evolution of Galaxies, 1016, 427

Otsuka, M., Izumiura, H., Tajitsu, A., \& Hyung, S. 2008b, ApJ, 682, L105

Sharpee, B. et al. 2007, ApJ, 659, 1265

Sterling, N. C. \& Dinerstein, H. L. 2008, ApJS, 174, 158

Suda, T. et al. 2008, PASJ, 60, 1159 